Research Highlight

\title{
Soft microsystems
}

\author{
Nam-Trung Nguyen* \\ Queensland Micro- and Nanotechnology Centre, Griffith University, Brisbane, \\ Queensland 4111, Australia
}

\footnotetext{
*Address correspondence to this author at the Queensland Micro- and Nanotechnology Centre, Griffith University, Brisbane, Queensland 4111, Australia; Tel: +61 (07) 373 53921; Fax: (+61 07) 373 58021;

E-mail: nam-trung.nguyen@griffith.edu.au
}

\begin{abstract}
Conventional microsystems including sensors and actuators are made of rigid materials such as silicon because of their origin from microelectronics. However, most micro- and nanosytems found in the nature are made of soft materials allowing them to deform depending on the task and external environment. The deformability allows natural microsystems to exceed the performance of their rigid micromachined counterparts. Inspired by nature, recent research efforts have been focused on soft microsystems including sensors, actuators and microfluidic platforms, promising a completely new research field with a broad range of applications.
\end{abstract}

Biomimetics or biomimicry has been evolved as a research field on systems, elements or materials that imitate their natural counterparts. Natural selection over millions of years provide an optimization process for engineering problems of biological systems. Imitation of nature in different length scales provides interesting solution to current engineering challenges. Soft robotics for instance is a branch of biomimetics that explores the concept of robots made of soft and compliant materials [1]. The Young's modulus of tissues in living systems are 3 to 5 orders of magnitude lower than that of metals, plastics or semiconductors that are used in conventional engineered systems. Recently, Rus and Tolley [1] reported the state of the art of soft robotics and discussed current solutions and challenges in actuation, sensing, fabrication and control of soft robots. Soft microsystems are smaller in size, they share the same challenges with soft robotics. However, the smaller size present unique opportunities in design of soft microsystems and in interfacing with biological systems.

Because of the soft material for making these systems, the most common actuation concepts are pneumatic or hydraulic schemes. The technology allows for the transfer of the silicene layer to a more useful substrate such as silicon. The process starts with the epitaxial growth of silicene on a thin $\mathrm{Ag}(111)$ layer deposited on a mica substrate, Fig. 1(a). The silicene layer was then protected by a 5-nm thick layer of alumina $\left(\mathrm{Al}_{2} \mathrm{O}_{3}\right)$, Fig. 1(b). The silicene layer sandwiched between and protected by the silver and alumina layers could be then detached from the mica substrate, Fig. 1(c). The alumina/silicence/silver film was than flipped upside down and transferred to a conventional oxidized silicon substrate, Fig. 1(d,e). The silicon acts as the gate electrode, while the silver layer was etched to form drain and source electrodes of the transistor, Fig. 1(f). To prevent the oxidization of the silicene layer, the authors developed a gentle potassium iodine-based etchant. Although the exposed silicene and the transistor have a lifetime of few minutes, the device has demonstrated the characteristics of a truly nanoelectronic transistor. 
In the near future, protecting the exposed silicene with another layer of more stable material such as Teflon could extend the life of the transistor. Making multi-layer silicences and allowing the upper layer to be oxidized could also protect the silicene working as semiconductor for the transistor. This proof-of-concept device may open up a new research field and probably a new industry of nanoelectronics.

Figure 1. Fabrication steps of the silicene transistor

\section{References}

[1] D. Rus, M. T. Tolley, Design, Fabrication and control of soft robots, Nature, 2015, 521(5934), 467-475.

[2] Y. Huang, N.T. Nguyen, K.S. Lok, P.P.F. Lee, M. Su, M. Wu, L. Kocgozlu, B. Ladoux, Multiarray cell stretching platform for high magnification real-time imaging, Nanomedicine, Nanomedicine, 2013, 8 (4), 543-553.

[3] G. G. Guzman-Verri, L.C.L.Y. Voon, Electronics structure of silicon-based nanostructures, Physical Review B, 2007, 76(7), 075131

[4] B. Aufray, A. Kara, S. Vizzini, H. Oughaddou, C. Leandri, B. Ealet, G. Le Lay, Graphene-like silicon nanoribbons on $\mathrm{Ag}(110)$ : A possible formation of silicene, Applied Physics Letters, 2010, 96(18), 183102.

[5] P. Vogt, P. De Padova, C. Quaresima, J. Avila, E. Frantzeskakis, M.C. Asensio, A. Resta, B. Ealet, G. Le Lay, Silicene: Compelling experimental evidence for graphenelike two-dimensional silicon, Physical Review Letters, 2012, 108(15), 155501.

[6] L. Tao, E. Cinquanta, D. Chiappe, C. Grazianetti, M. Fanciulli, M. Dubey, A. Molle, D. Akinwande, Silicene field-effect transistors operating at room temperature, Nature Nanotechnology, 2015, DOI: 10.103/NNANO.2014.325 